\title{
Gerechtigkeit in der Ressourcenverteilung bei Psychotherapie
}

\author{
Winfried Rief \\ Klinische Psychologie und Psychotherapie, Psychotherapie-Ambulanz, Philipps-Universität Marburg, Deutschland
}

Gerechtigkeit ist neben Autonomie, Unversehrtheit und Nutzenmehrung eines von 4 ethischen Grundprinzipien, die nicht nur bei wissenschaftlichen Studien, sondern auch bei Versorgungsfragen im Gesundheitssystem von Relevanz sind und beachtet werden sollten [Council for International Organizations of Medical Sciences (CIOMS) in collaboration with the World Health Organization (WHO), 2002]. Dies gilt im Besonderen, wenn es sich wie bei der Psychotherapie um eine Dienstleistung handelt, die über die Solidargemeinschaft der Krankenversicherten finanziert wird. Werden die Gelder der Krankenkassen zur Behandlung von Personen mit psychischen Erkrankungen gerecht verteilt? Setzen Psychotherapeuten ihre Arbeitskraft zur Behandlung von Menschen mit psychischen Erkrankungen gerecht ein? An welchen Stellen ergeben sich Hinweise, dass das Prinzip Gerechtigkeit gegebenenfalls nicht ausreichend im Gesundheitssystem berücksichtigt wird?

Grundsätzlich ist festzuhalten, dass viele Menschen, die Psychotherapie erhalten möchten, diese nicht bekommen. Die Mehrzahl der Menschen mit psychischen Problemen mit Krankheitswert würde Psychotherapie als Behandlungsform präferieren, erhält jedoch entweder keine Behandlung oder Pharmakotherapie [Rief et al., 2005]. Nur 6\% der Personen mit Depressionen erhält eine Psychotherapie [Mitteilung der KV Bayern, 2007]. Obwohl es wissenschaftlich keine Präferenz für Pharmakotherapie versus Psychotherapie gibt, erhalten offensichtlich selektiv viele Personen primär pharmakologische Behandlungsempfehlungen, und der Weg zur Psychotherapie bleibt ihnen versperrt. Ist bereits diese Tatsache unter dem Aspekt der Gerechtigkeit bedenklich, so stellt sich umso mehr die Frage, ob die Selektion der Personen, die es zum Psychotherapeuten schaffen, nach gerechten und bedarfsorientierten Regeln erfolgt.

In der zurzeit in England laufenden groß angelegten Studie «Improving Access to Psychological Therapy» wurde festge- stellt, dass gerade auch vorgeschriebene Überweisungsprozeduren im Gesundheitswesen zu einer sozialen Verschiebung der Ressourcenallokation führen. Sollten die Hausärzte die «Gatekeeper-Funktion» übernehmen und Patienten zur Psychotherapie zuweisen, bei denen sie es für angezeigt empfinden, ergibt sich ein deutliches soziales Gefälle: Sie überweisen überproportional viele Menschen mit höherer sozialer Stellung und geringerer Beeinträchtigung in die Psychotherapie. Wird diese Gatekeeper-Funktion ausgeschaltet (z.B. durch Direktzugangsmöglichkeit von Patienten zu Psychotherapeuten), so fällt der soziale Schichtungsfaktor weg und es findet sich eine erwartungsgemäße Anzahl von Personen auch aus niedrigen sozialen Schichten in der Psychotherapie [Gyani et al., 2011].

Soziale Schichtungsfaktoren werden jedoch auch durch die Psychotherapeuten selbst verschärft. Akademiker und Personen mit höherem Bildungsabschluss erhalten durchschnittlich längere Psychotherapien als Personen mit niedrigem Bildungsabschluss [Walendzik et al., 2011]. Dies ist besonders bemerkenswert, da Personen mit höheren Bildungsabschlüssen oftmals bessere Behandlungsprognosen haben, leichter auch von kürzeren Interventionen profitieren könnten und günstigere soziale Rahmenbedingungen für eine erfolgreiche Intervention aufweisen. Trotzdem wird ihnen von der Ressource Psychotherapie ein überproportionaler Anteil zugewiesen.

Aber auch andere Personengruppen sind in der psychotherapeutischen Versorgung unterrepräsentiert. Obwohl z.B. Mütter mit Klein- und Vorschulkindern eher stärker psychisch belastet sind als andere Personengruppen, findet man sie in stationären Settings für psychotherapeutische Behandlungen seltener vertreten. Offensichtlich werden zu viele Hürden wahrgenommen, um entweder in Behandlung zu gehen und dabei das Kind oder die Kinder gut versorgt zu wissen, oder es gibt zu wenig Möglichkeiten in qualitativ hochwertigen Kliniken, Kinder mit in die stationäre Einrichtung nehmen zu können.

\section{KARGER}

Fax +497614520714

Information@Karger.de

www.karger.com
(C) 2011 S. Karger GmbH, Freiburg

1016-6262/11/0214-0225\$38.00/0

Accessible online at:

www.karger.com/ver
Prof. Dr. Winfried Rief

Klinische Psychologie und Psychotherapie, Psychotherapie-Ambulanz

Philipps-Universität Marburg

Gutenbergstraße 18, 35032 Marburg, Deutschland

Tel. +49 6421 282-3641, Fax -8604

rief@staff.uni-marburg.de 
Besonders auffällig ist auch die unerwartet niedrige Häufigkeit von älteren Menschen mit Psychotherapie. Die Entwicklung von psychotherapeutischen Behandlungsprogrammen für ältere Menschen hat erst im letzten Jahrzehnt an Dynamik gewonnen, so dass zwischenzeitlich auch Behandlungsansätze vorliegen, die spezifisch auf ältere Menschen zugeschnitten sind [Hautzinger und Welz, 2004]. Historisch gesehen wurde früher zu Unrecht vermutet, dass psychotherapeutische Behandlungen bei Menschen über 65 gegebenenfalls keinen Erfolg mehr zeigen könnten. Neuere Studienergebnisse widerlegen diese Annahme eindeutig. Auch gibt es bei älteren Menschen durchaus Interesse, über Gespräche mit eigenen psychischen Problemen besser zurechtzukommen. Werden ältere Menschen zu wenig auf die Möglichkeit einer psychotherapeutischen Behandlung hingewiesen? Erleben sie zu große Hürden bei der Aufnahme einer Psychotherapie? Gelingt es Psychotherapeuten zu wenig, sich auf die spezielle Erlebniswelt von älteren Menschen einzustellen? Hier sind noch viele Fragen zu klären, um auch älteren Menschen in ausreichendem Maße Zugang zu psychotherapeutischer Versorgung zu verschaffen.

Jedoch auch bei bestimmten Krankheitsgruppen findet man psychotherapeutische Unterversorgung. Personen mit geistiger Behinderung tun sich oftmals sehr schwer, Psychotherapeuten zu finden. Personen mit schizophrenen Erkrankungen werden von vielen Psychotherapeuten abgelehnt, obwohl neuere Ergebnisse eindeutig belegen, dass Psychotherapie bei Personen mit schizophrenen Erkrankungen ähnliche Effektstärken erbringen kann wie die pharmakologische Behandlung mit Antipsychotika [Lincoln und Rief, 2007]. Neueste Forschungsergebnisse weisen sogar darauf hin, dass positive Psychotherapieeffekte selbst bei Personen mit Schizophrenie erreicht werden können, die keinerlei pharmakologische Behandlung erhalten [Morrison et al., 2011]. Auch Personen mit bipolaren Störungen [Breit-Gabauer et al., 2010] findet man selten in der psychotherapeutischen Praxis, obwohl auch hier erfolgreiche Behandlungsprogramme vorgestellt wurden. Gleiches gilt für Personen mit neuropsychologischen Schädigungen sowie Demenzen [Exner et al., 2010].

Ein besonderes Problem stellt die Versorgung von Menschen mit körperlichen Erkrankungen dar, die eine zusätzliche verhaltensmedizinische/psychotherapeutische Betreuung benötigen. Obwohl psychologische Ansätze bei chronischen Schmerzpatienten dauerhaft die Lebensqualität verbessern und auch hier die Effektstärken psychologischer Schmerzbewältigung durchaus mit medikamentösen Behandlungen konkurrieren können, zum Teil sogar bessere Langzeiteffekte erbringen, erhält nur ein kleiner Bruchteil von Personen mit chronischen Schmerzsyndromen auch psychologische Behandlung. Ähnliches gilt für Krankheitsbilder, bei denen belegt ist, dass eine komorbide Depression die körperliche Grunderkrankung deutlich verschlechtert und die Behandelbarkeit erschwert, wie z.B. bei koronaren Herzerkrankungen, Diabetes mellitus, Personen nach Schlaganfall oder Krebs.
Bei vielen Behandlern scheint das Bewusstsein für die Dringlichkeit zu fehlen, in solchen Fällen eine adäquate Behandlung durchzuführen. Gleichzeitig stellt man bei sehr vielen Psychotherapeuten eine gewisse Zurückhaltung fest, verhaltensmedizinische Interventionen in der ambulanten Praxis anzubieten. Oftmals fehlen auch grundlegende Informationen über die komplexen psychologischen und körperlichen Interaktionen, die in solchen Fällen notwendig sind. Die Diskussion, in Ergänzung zu entsprechenden Fortbildungen in der Medizin auch für psychologische Psychotherapeuten eine Fortbildung in «Somatopsychologie», Verhaltensmedizin oder Ähnlichem anzubieten, muss deshalb mit entsprechender Energie fortgesetzt werden bzw. es müssen Alternativen entwickelt werden, um die aktuelle Versorgungssituation für diesen Personenkreis zu verbessern.

Besonders schwierig stellt sich auch das Problem der Behandlung von «Tätern» dar. Wenn im Pädophilen-Projekt der Charité als ein Ergebnis berichtet wird, dass 60\% der Patienten zuvor erfolglos versuchten, einen ambulanten Psychotherapieplatz zu finden (Mitteilung auf der Projekt-Homepage: www.kein-taeter-werden.de), dann hat an dieser Stelle auch das System der psychotherapeutischen Versorgung versagt. Dies bedeutet, dass auch viele potentielle Täter Hilfesuchende sind, aber keine Hilfsangebote bekommen. Welches Potential zur Vermeidung von sexueller Gewalt [Trunk, 2010] und Kindesmissbrauch wohl in einer Verbesserung des psychotherapeutischen Versorgungsangebots für potentielle Täter liegt?

Wenn auf der einen Seite bestimmte Krankheitsgruppen unterrepräsentiert sind, bedeutet dies, dass auf der anderen Seite andere Krankheitsgruppen eher überrepräsentiert sind. So findet man in der ambulanten psychotherapeutischen Praxis überzufällig häufig die Diagnose «Anpassungsstörung» vor. Bedenkt man das Definitionskriterium, dem entsprechend diese Personen nicht außergewöhnlichen Belastungen ausgesetzt sind, sondern Belastungen, die eher typisch, wenn auch unangenehm im menschlichen Lebenslauf vorkommen, so erscheint die Priorisierung dieser Patientengruppe in der ambulanten psychotherapeutischen Versorgung schwer zu rechtfertigen. Hätte hier nicht wirklich der eine oder andere Patient auch das Potential, mit der Zeit selbständig mit den Problemen fertig zu werden? Zumindest stellt sich diese Frage bei Personen mit schizophrenen Erkrankungen, chronischen und schweren Depressionen, chronifizierten Essstörungen oder Ähnlichem nicht in dieser Form.

Selektionseffekte und damit auch Aspekte der Ungerechtigkeit werden zurzeit durch lange Wartezeiten sowohl im ambulanten als auch im stationären Bereich der psychotherapeutischen Versorgung verschärft. Wenn Kliniken und ambulante Psychotherapeuten ihre Patienten «handverlesen» können, kommen auch andere Entscheidungskriterien zum Zuge, die weniger die Behandlungsbedürftigkeit in den Vordergrund stellen. Gleichzeitig fehlt ein Anreizsystem, bei den Patienten, die es zum Psychotherapeuten geschafft haben, auch sparsam mit der Ressource Psychotherapie umzugehen. So muss ange- 
zweifelt werden, ob bei den vielen in Deutschland durchgeführten Langzeittherapien wirklich auch noch bei höheren Sitzungszahlen das primäre Ziel die Behandlung einer Krankheit ist und es sich nicht um allgemeine Selbsterfahrung, Lebenshilfe oder nur noch um stützende Einzelgespräche handelt. Der Autor ist sich sehr wohl bewusst, dass in vielen Fällen auch aus präventiven Gründen solche Maßnahmen indiziert sind, aber ist dies wirklich bei allen der Fall? Für andere Versorgungsfragen, z.B. aus dem Bereich Verhaltensmedizin, müssten auch neben der üblichen Dreiteilung in probatorische Sitzung, Kurzzeit-Psychotherapie und LangzeitPsychotherapie andere, attraktive Formate definiert werden, da viele verhaltensmedizinische Interventionen auf kürzere Sitzungszahlen zugeschnitten sind (5-12 Sitzungen). Gegebenenfalls kann auch diese Frage im Kontext einer spezifischen Weiterbildung «Somatopsychologie» thematisiert werden.

Warum ist es wichtig, dass die Ressourcenverteilung in unserem Gesundheitssystem auch dem Aspekt der Gerech- tigkeit folgt? Wir haben in Deutschland ein Gesundheitssystem, das Psychotherapie auf einem hohen Niveau integriert hat. Dies ist gerechtfertigt und die Notwendigkeit einer guten therapeutischen Versorgung ist ausreichend durch wissenschaftliche Studien untermauert. Allerdings geraten Versorgungssysteme unter Druck, wenn sie nicht als gerecht wahrgenommen werden. Deshalb ist die Reduktion von Ungerechtigkeit in der Ressourcenverteilung eine grundsätzliche Maßnahme zur Sicherung der Nachhaltigkeit einer psychotherapeutischen Versorgung. Aber nicht nur aus solchen ökonomischen Überlegungen sollte Verteilungsgerechtigkeit ein Ziel sein, das wir alle verfolgen: Auch zum Erhalt ethischer Werte in der Gesellschaft ist eine Reflexion auf individueller Ebene, aber auch auf der Versorgungsebene notwendig, wo Verletzungen des Gerechtigkeitsprinzips stattfinden. Gerechtigkeit ist ein definierender Wert unserer Gesellschaft und sollte auch für die psychotherapeutische Versorgung gelten.

\section{Literatur}

Breit-Gabauer B, Berg A, Demelbauer S, Schrott A,

Stampfer I, Lenz G: Frühwarnsymptome und Copingstrategien bei Patienten mit bipolaren Störungen. Verhaltenstherapie 2010;20:183-191.

Council for International Organizations of Medical Sciences (CIOMS) in collaboration with the World Health Organization (WHO): International Ethical Guidelines for Biomedical Research Involving Human Subjects. Genf, WHO, 2002.

Exner C, Döring B, Conradt N, Rief W: Integration von Verhaltenstherapie und Neuropsychologie. Vorstellung eines ambulanten Behandlungsansatzes für kognitive und emotional-motivationale Störungen nach erworbenen Hirnschädigungen. Verhaltenstherapie 2010;20:119-126.
Gyani A, Shafran R, Layard R, Clark DM: Enhancing Recovery Rates in IAPT Services: Lessons from Analysis of the Year One Data. www.iapt.nhs.uk/ downloads, accessed on October 27, 2011.

Hautzinger M, Welz S: Kognitive Verhaltenstherapie

bei Depressionen im Alter. Z Gerontol Geriatr 2004;37:427-435.

Lincoln T, Rief W: Kognitive Verhaltenstherapie von

Wahn und Halluzinationen. Z Klin Psychol Psychother 2007;36:164-175.

Morrison AP, Hutton P, Wardle M, Spencer H, Barratt S, Brabban A, Callcott P, Christodoulides T, Dudley R, French P, Lumley V, Tai SJ, Turkington D: Cognitive therapy for people with a schizophrenia spectrum diagnosis not taking antipsychotic medication: an exploratory trial. Psychol Med 2011; DOI: $10.1017 /$ S0033291711001899.
Rief W, Martin A, Klaiberg A, Brähler E: Specific effects of depression, panic, and somatic symptoms on illness behavior. Psychosom Med 2005;67:596-601.

Trunk D: Kindesmisshandlung: ein Abriss. Verhaltenstherapie 2010;20:11-18.

Walendzik A, Rabe-Menssen C, Lux G, Wasem J, Jahn R: Erhebung zur ambulanten psychotherapeutischen Versorgung 2010. Berlin, Deutsche Psychotherapeuten-Vereinigung, 2011. 